\title{
CENTERED BODIES AND DUAL MIXED VOLUMES
}

\author{
GAOYONG ZHANG
}

\begin{abstract}
We establish a number of characterizations and inequalities for intersection bodies, polar projection bodies and curvature images of projection bodies in $\mathbf{R}^{n}$ by using dual mixed volumes. One of the inequalities is between the dual Quermassintegrals of centered bodies and the dual Quermassintegrals of their central $(n-1)$-slices. It implies Lutwak's affirmative answer to the Busemann-Petty problem when the body with the smaller sections is an intersection body. We introduce and study the intersection body of order $i$ of a star body, which is dual to the projection body of order $i$ of a convex body. We show that every sufficiently smooth centered body is a generalized intersection body. We discuss a type of selfadjoint elliptic differential operator associated with a convex body. These operators give the openness property of the class of curvature functions of convex bodies. They also give an existence theorem related to a well-known uniqueness theorem about deformations of convex hypersurfaces in global differential geometry.
\end{abstract}

\section{INTRODUCTION}

Let $\mathbf{R}^{n}$ be the $n$-dimensional Euclidean space. A convex body $K \subset \mathbf{R}^{n}$ is a compact convex subset with nonempty interior. Let $\mathscr{K}$ denote the set of convex bodies in $\mathbf{R}^{n}$, and let $\mathscr{K}_{e}$ denote the symmetric convex bodies with respect to the origin. As Minkowski noted in the case of $\mathbf{R}^{3}$, the $(n-1)$ dimensional volume of the image of the orthogonal projection of $K \in \mathscr{K}$ onto a $(n-1)$-subspace is the support function of another convex body $\Pi K$, called the projection body of $K$. Projection bodies have received considerable attention in recent years (see, for example, $[4,11,16,24,36,38,39,45,47])$. Instead of considering the projection of a convex body, Busemann [6] proved that the $(n-1)$-dimensional volume of the intersection of $K \in \mathscr{K}_{e}$ with a $(n-1)$ subspace of $R^{n}$ is the radial function of another convex body $I K$, called by Lutwak the intersection body of $K$. The intersection body, which may be viewed as the dual of the projection body, is an important tool in understanding the sections of convex bodies. Lutwak studied intersection bodies by his dual mixed volumes. He defined intersection bodies for star bodies and related them to the spherical Radon transform. Many analogous theorems to those on projection bodies and mixed volumes were proved, see [25-28]. A slight extension of the definition of intersection bodies was given by using measures (see [18]). It states

Received by the editors May 6, 1992 and, in revised form, December 15, 1993.

1991 Mathematics Subject Classification. Primary 52A20, 52A40, 53C45.

Key words and phrases. Convex body, star body, intersection body, dual mixed volume, geometric inequality, curvature function. 
that a centered body $K$ is an intersection body if the inverse spherical Radon transform of the radial function of $K$ is a measure. Another slight extension of the original definition is to introduce the intersection body of order $i$ of a star body by using Lutwak's dual Quermassintegrals (see (2.4)). This is dual to the projection body of order $i$ of a convex body. We will work with these extended definitions and give characterizations for the intersection bodies by using dual mixed volumes (Theorems 2.12 and 2.15). These characterizations are related to the Busemann-Petty problem: If $K, L \in \mathscr{K}_{e}$, and $I K \subseteq I L$, does it follow that the volumes of $K$ and $L$ satisfy $V(K) \leq V(L)$ ? The relation says that the existence of nonintersection convex bodies is equivalent to a negative answer to the Busemann-Petty problem (see [13, 47]). Our characterizations for intersection bodies can be considered as duals of some well-known characterizations for projection bodies (see [16, 38, 39, 45, 46]). Lutwak [25] showed that the Busemann-Petty problem has an affirmative answer if $K$ is an intersection body. This result is implied by a geometric inequality about intersection bodies (Corollary 2.18). More general inequalities of intersection bodies are derived in Theorem 2.17. These inequalities are between the dual Quermassintegrals of centered bodies and the dual Quermassintegrals of their central ( $n-1)$-slices. They are related to the maximal slice conjecture (Corollary 2.19).

In the same spirit of studying intersection bodies, a number of characterizations and inequalities for polar projection bodies and the curvature image of projection bodies are established by dual mixed volumes in sections 3 and 4 . These involve centroid bodies and the affine surface area.

In section 5 we use $L^{p}$ estimates for the spherical Radon transform to show that every centered body in $\mathbf{R}^{n}$ of class $C^{k}, k=\left[\frac{n+1}{2}\right]-1$, is a generalized intersection body (Theorem 5.4). This implies that the class of generalized intersection bodies is dense in the class of centered bodies (Corollary 5.5). It is shown that the dual generating distribution has order at most $\left[\frac{n+1}{2}\right]-1$ (Theorem 5.6).

In section 6 we discuss a type of selfadjoint elliptic differential operator associated with a convex body. These operators are applied to show the openness of the class of curvature functions with Hölder continuity (Theorem 6.8). A consequence of the openness is a strengthening of Weil's theorem in [47] about the denseness of the difference of $j$ th surface area measures in the space $M_{e}$ of signed even measures (Corollary 6.11). Finally, we prove an existence theorem about deformations of convex hypersurfaces, which is a companion to a well-known uniqueness theorem in global differential geometry (Theorem 6.14).

I am very grateful to Professor E. Lutwak for valuable references and conversations during his visit to Temple University. I would like to express my thanks to Professor E. Grinberg for his constant encouragement and help. I also would like to thank Professor P. Goodey and the referee for many helpful comments.

\section{Preliminaries}

Let $S^{n-1}$ be the unit sphere in $\mathbf{R}^{n}$. For a compact set $K$ in $\mathbf{R}^{n}$ which is star shaped with respect to the origin, define the radial function $\rho_{K}$ of $K$ by

$$
\rho_{K}(u)=\max \{\lambda \geq 0: \lambda u \in K\} \quad \text { for } u \in S^{n-1} \text {. }
$$

If $\rho_{K}$ is continuous we shall call $K$ a star body. A star body which is centrally symmetric with respect to the origin will be called a centered body. We shall use 
$\mathscr{S}$, and $\mathscr{S}_{e}$ to denote respectively the set of star bodies and the set of centered bodies. Obviously, for $K, L \in \mathscr{S}$,

$$
K \subseteq L \text { if and only if } \rho_{K} \leq \rho_{L} .
$$

Hence, a star body is uniquely determined by its radial function.

Associated with a convex body $K$ is its support function $h_{K}$ defined on $\mathbf{R}^{n}$ by

$$
h_{K}(x)=\max \{\langle x, y\rangle: y \in K\},
$$

where $\langle x, y\rangle$ is the usual inner product of $x$ and $y$ in $\mathbf{R}^{n}$. The support function $h_{K}$ is positively homogeneous of degree 1 . We will usually be concerned with the restriction of the support function to the unit sphere $S^{n-1}$.

If $K_{1}, \cdots, K_{r} \in \mathscr{K}$ and $\lambda_{1}, \cdots, \lambda_{r} \geq 0$, then the Minkowski linear combination is

$$
\lambda_{1} K_{1}+\cdots+\lambda_{r} K_{r}=\left\{\lambda_{1} x_{1}+\cdots+\lambda_{r} x_{r}: x_{i} \in K_{i}\right\} .
$$

Note that if $K, L \in \mathscr{K}$ and $\alpha, \beta \geq 0$, then

$$
h_{\alpha K+\beta L}=\alpha h_{K}+\beta h_{L}
$$

and

$$
K \subseteq L \text { if and only if } h_{K} \leq h_{L} .
$$

Hence, a convex body is uniquely determined by its support function.

Let $V(K)$ denote the volume of $K$. The volume of $\lambda_{1} K_{1}+\cdots+\lambda_{r} K_{r}$ is a homogeneous $n$ th-degree polynomial in the $\lambda_{i}$,

$$
V\left(\lambda_{1} K_{1}+\cdots+\lambda_{r} K_{r}\right)=\sum V\left(K_{i_{1}}, \cdots, K_{i_{n}}\right) \lambda_{i_{1}} \cdots \lambda_{i_{n}},
$$

where the sum is taken over all $n$-tuples $\left(i_{1}, \cdots, i_{n}\right)$ whose entries are positive integers not exceeding $r$. The coefficient $V\left(K_{i_{1}}, \cdots, K_{i_{n}}\right)$ is nonnegative and depends only on the bodies $K_{i_{1}}, \cdots, K_{i_{n}}$. It is called the mixed volume of $K_{i_{1}}, \cdots, K_{i_{n}}$. Let

$$
V_{i}(K, L)=V(\underbrace{K, \cdots, K}_{n-i \text { times }}, \underbrace{L, \cdots, L}_{i \text { times }}) .
$$

The Minkowski inequality states that if $K, L \in \mathscr{K}$, then

$$
V_{1}(K, L)^{n} \geq V(K)^{n-1} V(L)
$$

with equality if and only if $K$ and $L$ are homothetic.

Associated with the convex bodies $K_{1}, \cdots, K_{n-1} \in \mathscr{H}$ is a unique positive finite Borel measure on $S^{n-1}, S\left(K_{1}, \cdots, K_{n-1} ; \cdot\right)$, called the mixed surface area measure of $K_{1}, \cdots, K_{n-1}$, such that for any $K \in \mathscr{K}$ there is the integral representation (see, for example, [52, p. 275; 5, p. 64; 7, p. 166 and 10, p. 69])

$$
V\left(K_{1}, \cdots, K_{n-1}, K\right)=\frac{1}{n} \int_{u \in S^{n-1}} h_{K}(u) d S\left(K_{1}, \cdots, K_{n-1} ; u\right) .
$$

The mixed surface area measure has the following properties (see, for instance, $[24,(1.5)$ and (1.6)])

$$
\begin{aligned}
& S\left(K_{1}+L_{1}, K_{2}, \cdots, K_{n-1} ; \cdot\right) \\
& \quad=S\left(K_{1}, \cdots, K_{n-1} ; \cdot\right)+S\left(L_{1}, K_{2}, \cdots, K_{n-1} ; \cdot\right), \\
& S\left(\lambda_{1} K_{1}, \cdots, \lambda_{n-1} K_{n-1} ; \cdot\right)=\lambda_{1} \cdots \lambda_{n-1} S\left(K_{1}, \cdots, K_{n-1} ; \cdot\right) .
\end{aligned}
$$


The surface area measure of order $j$ of $K$ is defined by

$$
S_{j}(K ; \cdot)=S(\underbrace{K, \cdots, K}_{j}, \underbrace{B, \cdots, B}_{n-j-1} ; \cdot),
$$

where $B$ is the unit ball in $\mathbf{R}^{n}$. In particular, $S_{n-1}(K ; \cdot)$ is called the surface area measure of $K$. Then by (1.2) we have

$$
V(L, \underbrace{K, \cdots, K}_{j}, \underbrace{B, \cdots, B}_{n-j-1})=\frac{1}{n} \int_{S^{n-1}} h_{L}(u) d S_{j}(K ; u)
$$

for all $K, L \in \mathscr{K}$ and $1 \leq j \leq n-1$.

A convex body $K \in \mathscr{K}$ is said to have a continuous positive curvature function of order $j, f_{j}(K ; \cdot): S^{n-1} \longrightarrow(0, \infty)$, if for each $L \in \mathscr{K}$, we have

$$
V(L, \underbrace{K, \cdots, K}_{j}, \underbrace{B, \cdots, B}_{n-j-1})=\frac{1}{n} \int_{S^{n-1}} h_{L}(u) f_{j}(K ; u) d S_{n-1}(B ; u) .
$$

If the boundary $\partial K$ is of class $C^{2}$ and has positive Gaussian curvature, then $f_{j}(K ; \cdot)$ is the $j$ th elementary symmetric function of the radii of principal curvature. Denote by $\mathscr{F}$ the class of convex bodies which have positive curvature functions of order $n-1$. Let $f_{K}=f_{n-1}(K ; \cdot)$. We call it the curvature function of $K$.

The projection body of $K, \Pi K$, is defined by

$$
h_{\Pi K}(u)=\frac{1}{2} \int_{S^{n-1}}|\langle u, x\rangle| d S_{n-1}(K ; x) .
$$

A body $K \in \mathscr{K}_{e}$ is called a zonoid if its support function $h_{K}$ can be expressed as

$$
h_{K}(u)=\frac{1}{2} \int_{S^{n-1}}|\langle u, x\rangle| d \mu_{K}(x),
$$

with an even positive finite Borel measure $\mu_{K}$ on $S^{n-1}$. The measure $\mu_{K}$ is uniquely determined by $K$, and called the generating measure of $K$. In view of (1.6), a projection body is a zonoid. Conversely, every centered zonoid is a projection body (see $[39, \S 10]$ ). Denote by $\mathscr{Z}$ the set of projection bodies (centered zonoids).

We follow Lutwak $[25,26]$ to define the dual mixed volumes. If $x_{1}, \cdots, x_{r}$ $\in \mathbf{R}^{n}$, then $x_{1} \tilde{f} \cdots \tilde{+} x_{r}$ is defined to be the usual vector sum of $x_{1}, \cdots, x_{r}$, provided $x_{1}, \cdots, x_{r}$ all lie in a 1-dimensional subspace of $\mathbf{R}^{n}$, and as the zero vector otherwise. If $K_{1}, \cdots, K_{r} \in \mathscr{S}$ and $\lambda_{1}, \cdots, \lambda_{r} \in \mathbf{R}$, then the radial Minkowski linear combination, $\lambda_{1} K_{1} \tilde{+} \cdots \tilde{+} \lambda_{r} K_{r}$, is defined by

$$
\lambda_{1} K_{1} \tilde{+} \cdots \tilde{+} \lambda_{r} K_{r}=\left\{\lambda_{1} x_{1} \tilde{+} \cdots \tilde{+} \lambda_{r} x_{r}: x_{i} \in K_{i}\right\} .
$$

We note that for $K, L \in \mathscr{S}$ and $\alpha, \beta \geq 0$,

$$
\rho_{\alpha K \dot{+} \beta L}=\alpha \rho_{K}+\beta \rho_{L} \text {. }
$$

From this, one easily shows that for $K_{1}, \cdots, K_{r} \in \mathscr{S}$, and $\lambda_{1}, \cdots, \lambda_{r} \geq 0$, the volume of the radial Minkowski combination $\lambda_{1} K_{1} \tilde{+} \cdots \tilde{+} \lambda_{r} K_{r}$ is a homogeneous $n$ th-degree polynomial in the $\lambda_{i}$,

$$
V\left(\lambda_{1} K_{1} \tilde{+} \cdots \tilde{+} \lambda_{r} K_{r}\right)=\sum \tilde{V}_{i_{1} \cdots i_{n}} \lambda_{i_{1}} \cdots \lambda_{i_{n}},
$$


where the sum is taken over all $n$-tuples $\left(i_{1}, \cdots, i_{n}\right)$ whose entries are positive integers not exceeding $r$. The coefficient $\tilde{V}_{i_{1} \cdots i_{n}}$ is nonnegative and depends only on the bodies $K_{i_{1}}, \cdots, K_{i_{n}}$. It is written as $\tilde{V}\left(K_{i_{1}}, \cdots, K_{i_{n}}\right)$ and is called the dual mixed volume of $K_{i_{1}}, \cdots, K_{i_{n}}$. For simplicity, let $d u=d S_{n-1}(B ; u)$ be the volume element of $S^{n-1}$. We have the following integral representation of dual mixed volumes:

$$
\tilde{V}\left(K_{1}, \cdots, K_{n}\right)=\frac{1}{n} \int_{S^{n-1}} \rho_{K_{1}}(u) \cdots \rho_{K_{n}}(u) d u .
$$

The $i$ th dual mixed volumes of $K$ and $L$ is

$$
\tilde{V}_{i}(K, L)=\frac{1}{n} \int_{S^{n-1}} \rho_{K}(u)^{n-i} \rho_{L}(u)^{i} d u,
$$

where $i$ may be any real number. In particular, we use $i=-1$.

The dual Minkowski inequalities are (see [25, (4.2), 26, (5.5)])

$$
\begin{aligned}
\tilde{V}_{1}(K, L)^{n} & \leq V(K)^{n-1} V(L), \\
\tilde{V}_{-1}(K, L)^{n} & \geq V(K)^{n+1} V(L)^{-1} .
\end{aligned}
$$

Each inequality holds with equality if and only if $K$ and $L$ are dilations of each other.

We will use some spaces of functions. Denote by $C^{k}\left(S^{n-1}\right)(k=0,1,2, \cdots)$ the space of real functions on $S^{n-1}$ whose $k$ th derivatives are continuous, and $C_{e}^{k}\left(S^{n-1}\right)$ the subspace of even functions in $C^{k}\left(S^{n-1}\right)$. Let $C^{k, \alpha}\left(S^{n-1}\right)$ $(0<\alpha<1)$ be the space of functions in $C^{k}\left(S^{n-1}\right)$ whose $k$ th derivatives are Hölder continuous of order $\alpha$, and let $C_{e}^{k, \alpha}\left(S^{n-1}\right)$ be the subspace of even functions in $C^{k, \alpha}\left(S^{n-1}\right)$. All of these spaces are Banach spaces with the usual uniform norms (see $\left[15, \S 4.1 ; 1\right.$, p. 36]). For $f, g \in C\left(S^{n-1}\right)=C^{0}\left(S^{n-1}\right)$, define $(f, g)$ by

$$
(f, g)=\int_{S^{n-1}} f(u) g(u) d u .
$$

Let $C_{e}^{\infty}\left(S^{n-1}\right)$ be the space of infinitely differentiable even functions on $S^{n-1}$. Assume that $C_{e}^{\infty}\left(S^{n-1}\right)$ is endowed with the topology of uniform convergence of all derivatives. Let $\mathscr{D}_{e}\left(S^{n-1}\right)$ be the space of even distributions on $S^{n-1}$; this is the dual of the space $C_{e}^{\infty}\left(S^{n-1}\right)$. Denote by $\mathscr{M}_{e}$ the space of even signed finite measures on $S^{n-1}$, which is the dual space of $C_{e}\left(S^{n-1}\right)$. Finally, let

$$
\mathscr{F}_{e}^{k, \alpha}=\left\{K \in \mathscr{F}: h_{K} \in C_{e}^{k, \alpha}\left(S^{n-1}\right)\right\} \text {. }
$$

Note that for $k \geq 2$ we have

$$
\mathscr{F}_{e}^{k, \alpha}=\left\{K \in \mathscr{F}: \rho_{K} \in C_{e}^{k, \alpha}\left(S^{n-1}\right)\right\} ;
$$

see [21, Part IV].

\section{Characterizations AND INEQUalities OF INTERSECTION BODIES}

Let us start with the basic definition. The intersection body IK of a star body $K \in \mathscr{S}$ is defined as the centered body whose radial function is given by

$$
\rho_{I K}(u)=\operatorname{vol}_{n-1}\left(K \cap u^{\perp}\right) \quad \text { for } u \in S^{n-1},
$$


where $u^{\perp}$ is the $(n-1)$-dimensional subspace of $\mathbf{R}^{n}$ orthogonal to $u$ (see Lutwak [25]). We will consider some generalizations of this definition.

Lutwak introduced the dual Quermassintegrals of star bodies which are given by (see [25])

$$
\tilde{W}_{n-i}(K)=\tilde{V}_{n-i}(K, B)=\frac{1}{n} \int_{S^{n-1}} \rho_{K}^{i}(u) d u
$$

for $0<i<n$, and $\tilde{W}_{0}(K)=V(K), \tilde{W}_{n}(K)=V(B)=\kappa_{n}$. Denote by $\operatorname{vol}_{i}(\cdot)$ the $i$-dimensional volume. The importance of the dual Quermassintegrals lies in the fact that the $(n-i)$ th dual Quermassintegral of a star body $K$ is proportional to the mean of the $i$-dimensional volumes of the slices of $K$ by the $i$-dimensional subspaces of $\mathbf{R}^{n}$, that is (see [25, (2.12)]),

$$
\tilde{W}_{n-i}(K)=\frac{\kappa_{n}}{\kappa_{i}} \int_{G(n, i)} \operatorname{vol}_{i}(K \cap \xi) d \mu_{i}(\xi),
$$

where $G(n, i)$ is the Grassmann manifold of $i$-dimensional subspaces of $\mathbf{R}^{n}$, and $\mu_{i}$ the Haar measure on $G(n, i)$, normalized by $\mu_{i}(G(n, i))=1$. For $K \in \mathscr{S}$, the intersection $K \cap u^{\perp}$ is a star body in $(n-1)$-dimensional space. Let $\tilde{\mathbf{w}}_{n-1-i}\left(K \cap u^{\perp}\right)$ be the $(n-1-i)$ th dual Quermassintegral of $K \cap u^{\perp}$ in $\mathbf{R}^{n-1}$, which is called the dual $(n-1-i)$-girth of $K$ in the direction $u$ (see [25]).

The intersection body of order $i$ of a star body $K, I_{i} K$, is defined by

$$
\rho_{I_{i} K}(u)=\tilde{w}_{n-1-i}\left(K \cap u^{\perp}\right), \quad u \in S^{n-1} .
$$

Hence, $I K=I_{n-1} K$.

The intersection body of order $i$ is closely related to the spherical Radon transform. For $f \in C\left(S^{n-1}\right)$, the spherical Radon transform of $f, R f$, is defined by

$$
(R f)(u)=\frac{1}{n-1} \int_{S^{n-1} \cap u^{\perp}} f(v) d s(v),
$$

where $d s$ is the volume element of $S^{n-1} \cap u^{\perp}$. By applying the spherical Radon transform to the $i$ th power of the radial function of a star body, we have

$$
\begin{aligned}
\left(R \rho_{K}^{i}\right)(u) & =\frac{1}{n-1} \int_{S^{n-1} \cap u^{\perp}} \rho_{K}^{i}(v) d s(v) \\
& =\frac{\kappa_{n-1}}{i \kappa_{i}} \int_{G(n-1, i)} \int_{S^{n-2} \cap \xi} \rho_{K}^{i}(v) d S_{i-1}(B ; v) d \mu_{i}(\xi) \\
& =\frac{\kappa_{n-1}}{\kappa_{i}} \int_{G(n-1, i)} \operatorname{vol}_{i}(K \cap \xi) d \mu_{i}(\xi) .
\end{aligned}
$$

Hence, (2.3) and (2.4) give

$$
\rho_{I_{i} K}(u)=\left(R \rho_{K}^{i}\right)(u)=\tilde{\mathbf{w}}_{n-1-i}\left(K \cap u^{\perp}\right) .
$$

When restricted to $C_{e}^{\infty}\left(S^{n-1}\right)$, the spherical Radon transform $R: C_{e}^{\infty}\left(S^{n-1}\right) \rightarrow$ $C_{e}^{\infty}\left(S^{n-1}\right)$ is a continuous bijection (see Helgason [22, p. 161]). It is also selfadjoint, i.e., for $f, g \in C\left(S^{n-1}\right)$,

$$
(f, R g)=(R f, g) \text {. }
$$


From the injectivity of $R$ and (2.6), we conclude the result: If $K, L \in \mathscr{S}_{e}$, then $I_{i} K=I_{i} L$ if and only if $K=L$. This is the counterpart of Chakerian's uniqueness theorem about projection bodies of order $i$ (see [11, Theorem 1; 39, Theorem 10.2]).

As observed by Goodey and Weil [17, p. 677], $R$ is a continuous bijection of $\mathscr{D}_{e}\left(S^{n-1}\right)$ to itself. For $K \in \mathscr{S}_{e}$, we call the distribution $R^{-1} \rho_{K}$ the dual generating distribution of $K$, denoted by $\tilde{\mu}_{K}$. A body $K \in \mathscr{S}_{e}$ is called an intersection body if the dual generating distribution $\tilde{\mu}_{K}$ is a measure. Let $\mathscr{I}$ denote the set of intersection bodies. A body $K \in \mathscr{S}_{e}$ is called a generalized intersection body if the dual generating distribution $\tilde{\mu}_{K}$ is a signed measure. These definitions of intersection body and generalized intersection body are dual to the concepts of zonoids and generalized zonoids (see [18]). It is clear that the $i$ th intersection body $I_{i} K$ of a star body $K$ is an intersection body. We will show (in $\S 5$ ) that a sufficiently smooth intersection body is the intersection body of a centered body.

In the following, we will use the notation

$$
\tilde{V}(K, i ; L, j ; C)=\tilde{V}(\underbrace{K, \cdots, K}_{i}, \underbrace{L, \cdots, L}_{j}, C_{i+j+1}, \cdots, C_{n}) \text {, }
$$

in particular,

$$
\tilde{V}(K, i ; L, 1 ; B)=\tilde{V}(\underbrace{K, \cdots, K}_{i}, L, \underbrace{B, \cdots, B}_{n-i-1}),
$$

where $B$ is the unit ball in $\mathbf{R}^{n}$. By using the Hölder inequality, we have the inequality (see [28, Theorem 1])

$$
\tilde{V}(K, i ; L, j ; C)^{i+j} \leq \tilde{V}(K, i+j ; C)^{i} \tilde{V}(L, i+j ; C)^{j}
$$

with equality if and only if $K$ and $L$ are dilations of each other. Inequality (2.8) can be viewed as the dual Alexandrov-Fenchel inequality (see [11, (2.2)], for the Alexandrov-Fenchel inequality).

Lemma 2.9. Let $K, L \in \mathscr{S}$. Then $I_{i} K \subseteq I_{i} L$ if and only if

$$
\tilde{V}(K, i ; N, 1 ; B) \leq \tilde{V}(L, i ; N, 1 ; B)
$$

for every $N \in \mathscr{I}$.

Proof. For $N \in \mathscr{I}$, let $\tilde{\mu}_{N}=R^{-1} \rho_{N}$ be the dual generating measure of $N$. Then, by (1.8) and (2.7),

$$
\begin{aligned}
n \tilde{V}(K, i ; & N, 1 ; B)-n \tilde{V}(L, i ; N, 1 ; B) \\
& =\left(\rho_{K}^{i}, \rho_{N}\right)-\left(\rho_{L}^{i}, \rho_{N}\right) \\
& =\left(R \rho_{K}^{i}, R^{-1} \rho_{N}\right)-\left(R \rho_{L}^{i}, R^{-1} \rho_{N}\right) \\
& =\int_{S^{n-1}}\left(\rho_{I_{i} K}(u)-\rho_{I_{i} L}(u)\right) d \tilde{\mu}_{N}(u) .
\end{aligned}
$$

This proves the lemma.

By the definition of dual generating distribution and (2.7), we have

$$
\tilde{\mu}_{K}(f)=\left(\rho_{K}, R^{-1} f\right), \quad f \in C_{e}^{\infty}\left(S^{n-1}\right) .
$$


The distribution $\tilde{\mu}_{K}$ is a measure if and only if $\tilde{\mu}_{K}(f) \geq 0$ for all $f \geq 0$, $f \in C_{e}^{\infty}\left(S^{n-1}\right)$, see [42]. Let $g=R^{-1} f$. Then (2.10) gives that a body $K \in \mathscr{S}_{e}$ is an intersection body if and only if

$$
\int_{S^{n-1}} \rho_{K}(u) g(u) d u \geq 0 \text { whenever } R g \geq 0, g \in C_{e}^{\infty}\left(S^{n-1}\right) .
$$

The following lemma is an extension of this. It was proved in [18] in a more abstract setting. For self-containedness, we include a proof here.

Lemma 2.11. Let $\mathcal{N}$ be a dense subset of $C_{e}\left(S^{n-1}\right)$. Then a body $K \in \mathscr{S}_{e}$ is an intersection body if and only if

$$
\int_{S^{n-1}} \rho_{K}(u) g(u) d u \geq 0 \text { whenever } R g \geq 0, g \in \mathscr{N} \text {. }
$$

Proof. Let $\mathscr{N}$ be any dense subset of $C_{e}\left(S^{n-1}\right)$. We show that the set $\{g \in$ $\mathscr{N}: R g \geq 0\}$ is dense in $\left\{g \in C_{e}\left(S^{n-1}\right): R g \geq 0\right\}$. It is enough to show $\{g \in \mathscr{N}: R g \geq 0\}$ is dense in $\left\{g \in C_{e}\left(S^{n-1}\right): R g>0\right\}$. Take $g \in\{g \in$ $\left.C_{e}\left(S^{n-1}\right): R g>0\right\}$. There are $g_{i} \in \mathscr{N}$ so that $g_{i} \rightarrow g$ in $C_{e}\left(S^{n-1}\right)$. We want to show $R g_{i} \geq 0$ for $i$ sufficiently large. Assume this is not true. Then there exists a sequence $\left\{x_{k}\right\}$ in $S^{n-1}$ which converges to a point $x \in S^{n-1}$ and a subsequence $\left\{g_{i_{k}}\right\}$ such that $R g_{i_{k}}\left(x_{k}\right)<0$. Since $R g \in C_{e}\left(S^{n-1}\right)$ and $R g_{i_{k}}$ converges to $R g$ in $C_{e}\left(S^{n-1}\right)$,

$0<R g(x)<R g(x)-R g_{i_{k}}\left(x_{k}\right)=R g(x)-R g\left(x_{k}\right)+R g\left(x_{k}\right)-R g_{i_{k}}\left(x_{k}\right) \longrightarrow 0$.

This is impossible. Therefore, the condition (2.12) is equivalent to

$$
\int_{S^{n-1}} \rho_{K}(u) g(u) d u \geq 0 \text { whenever } R g \geq 0, g \in C_{e}\left(S^{n-1}\right) .
$$

Since $C_{e}^{\infty}\left(S^{n-1}\right)$ is dense in $C_{e}\left(S^{n-1}\right)$, we conclude the lemma.

Henceforth, $\mathscr{P}$ always denotes a dense cone of $\mathscr{K}_{e}$. Let $i \in\{1,2, \cdots, n-$ $1\}$.

Theorem 2.13. Let $K \in \mathscr{S}_{e}$. If $K$ is an intersection body, then for all $L, M \in$ $\mathscr{S}_{e}$ there is the implication

$$
I_{i} L \subseteq I_{i} M \quad \Longrightarrow \quad \tilde{V}(L, i ; K, 1 ; B) \leq \tilde{V}(M, i ; K, 1 ; B) .
$$

Conversely, let $M \in \mathscr{F}_{e}^{2}$ be fixed. If the implication (2.14) holds for all $L \in \mathscr{P}$, then $K$ is an intersection body.

Proof. By Lemma 2.9, the necessity is clear. Let $M \in \mathscr{F}_{e}^{2}$ and $q \in C_{e}^{2}\left(S^{n-1}\right)$. Then $\rho_{M}-t q \in C_{e}^{2}\left(S^{n-1}\right)$ is positive for $t$ sufficiently small. Let $M_{t}$ be the centered body with radial function $\rho_{M}-t q$. The family of closed hypersurfaces

$$
\partial M_{\imath}: S^{n-1} \longrightarrow \mathbf{R}^{n}, \quad u \longrightarrow \rho_{M}(u) u-t q(u) u, \quad t \in(-\epsilon, \epsilon),
$$

is a deformation of $\partial M$. Note that $\partial M$ has strictly positive Gauss curvature. From the classical formula of Gauss curvature using local coordinates and the compactness of $S^{n-1}$, it is easy to see that the Gauss curvature of $\partial M_{t}$ is strictly positive for $t$ sufficiently small. Therefore, $M_{t} \in \mathscr{F}_{e}^{2}$ for $t$ sufficiently small. From the identity

$$
\rho_{M}^{i}-\left(\rho_{M}-t q\right)^{i}=i \rho_{M}^{i-1} q t+O\left(t^{2}\right)
$$


and the denseness of $C_{e}^{2}\left(S^{n-1}\right)$ in $C_{e}\left(S^{n-1}\right)$, we conclude that the set

$$
\mathscr{N}=\left\{c \rho_{M}^{i}-\rho_{L}^{i}: c>0, L \in \mathscr{K}_{e}\right\}
$$

is dense in $C_{e}\left(S^{n-1}\right)$. Let $g=c \rho_{M}^{i}-\rho_{L}^{i}$. Then

$$
c^{-1} \int_{S^{n-1}} \rho_{K}(u) g(u) d u=\tilde{V}(M, i ; K, 1 ; B)-\tilde{V}\left(c^{-1 / i} L, i ; K, 1 ; B\right) .
$$

From (2.6) we have

$$
c^{-1} R g=\rho_{I_{i} M}-\rho_{I_{i}\left(c^{-1 / i}\right)} \text {. }
$$

Therefore, (2.14) is equivalent to (2.12), and we obtain the theorem by applying Lemma 2.11 to the above $\mathscr{N}$.

Theorem 2.15. Let $K \in \mathscr{S}_{e}$. If $K$ is an intersection body, then for all $L, M \in$ $\mathscr{S}_{e}$

$$
\frac{\tilde{V}(L, i ; K, 1 ; B)}{\tilde{V}(M, i ; K, 1 ; B)} \leq \max _{u \in S^{n-1}} \frac{\tilde{w}_{n-1-i}\left(L \cap u^{\perp}\right)}{\tilde{w}_{n-1-i}\left(M \cap u^{\perp}\right)} .
$$

Conversely, let $M \in \mathscr{F}_{e}^{2}$ be fuxed. If the inequality (2.16) holds for all $L \in \mathscr{P}$, then $K$ is an intersection body.

Proof. Obviously, the inequality (2.16) implies the implication (2.14). Hence (2.16) is sufficient to guarantee that $K$ is an intersection body. If $K$ is an intersection body with dual generating measure $\tilde{\mu}_{K}$, then

$$
\begin{aligned}
\frac{\tilde{V}(L, i ; K, 1 ; B)}{\tilde{V}(M, i ; K, 1 ; B)} & =\frac{\left(\rho_{L}^{i}, \rho_{K}\right)}{\left(\rho_{M}^{i}, \rho_{K}\right)}=\frac{\left(R \rho_{L}^{i}, R^{-1} \rho_{K}\right)}{\left(R \rho_{M}^{i}, R^{-1} \rho_{K}\right)} \\
& =\frac{\int_{u \in S^{n-1}} \rho_{I_{i} L}(u) d \tilde{\mu}_{K}(u)}{\int_{u \in S^{n-1}} \rho_{I_{i} M}(u) d \tilde{\mu}_{K}(u)} \leq \max _{u \in S^{n-1}} \frac{\rho_{I_{i} L}(u)}{\rho_{I_{i} M}(u)} \\
& =\max _{u \in S^{n-1}} \frac{\tilde{\mathbf{w}}_{n-1-i}\left(L \cap u^{\perp}\right)}{\tilde{w}_{n-1-i}\left(M \cap u^{\perp}\right)} .
\end{aligned}
$$

Hence, (2.16) is necessary.

Theorem 2.17. If $K \in \mathscr{I}$ and $M \in \mathscr{S}_{e}$, then

$$
\left[\frac{\tilde{W}_{n-i-1}(K)}{\tilde{W}_{n-i-1}(M)}\right]^{\frac{1}{i+1}} \leq \max _{u \in S^{n-1}} \frac{\tilde{w}_{n-1-i}\left(K \cap u^{\perp}\right)}{\tilde{w}_{n-1-i}\left(M \cap u^{\perp}\right)}
$$

with equality if and only if $K$ and $M$ are dilations of each other. Proof. Let $L=K$ in (2.16). From (2.2) and (2.8) we have

$$
\frac{\tilde{V}(L, i ; K, 1 ; B)}{\tilde{V}(M, i ; K, 1 ; B)} \geq \frac{\tilde{W}_{n-i-1}(K)}{\tilde{W}_{n-i-1}(M)^{\frac{i}{i+1}} \tilde{W}_{n-i-1}(K)^{\frac{1}{i+1}}}=\left[\frac{\tilde{W}_{n-i-1}(K)}{\tilde{W}_{n-i-1}(M)}\right]^{\frac{i}{i+1}} .
$$

This proves the theorem.

Let $i=n-1$ in Theorem 2.17. We obtain 
Corollary 2.18. If $K \in \mathscr{I}$ and $M \in \mathscr{S}_{e}$, then

$$
\left[\frac{V(K)}{V(M)}\right]^{\frac{n-1}{n}} \leq \max _{u \in S^{n-1}} \frac{\operatorname{vol}_{n-1}\left(K \cap u^{\perp}\right)}{\operatorname{vol}_{n-1}\left(M \cap u^{\perp}\right)}
$$

with equality if and only if $K$ and $M$ are dilations of each other.

The above corollary implies that for $K \in \mathscr{F}$ and $M \in \mathscr{S}_{e}$ if

$$
\operatorname{vol}_{n-1}\left(K \cap u^{\perp}\right) \leq \operatorname{vol}_{n-1}\left(M \cap u^{\perp}\right) \text { for all } u \in S^{n-1}
$$

then

$$
V(K) \leq V(M) .
$$

This result was proved by Lutwak ([25, Theorem 10.1]), which is an affirmative answer to the Busemann-Petty problem if $K$ is an intersection body. On the other hand, if we choose $\lambda>0$ so that

$$
\max _{u \in S^{n-1}} \frac{\operatorname{vol}_{n-1}\left((\lambda K) \cap u^{\perp}\right)}{\operatorname{vol}_{n-1}\left(M \cap u^{\perp}\right)}=1,
$$

then Lutwak's result gives Corollary 2.18. Hence, Corollary 2.18 can be viewed as a reinterpretation of Lutwak's result. By taking $M=B$ in Corollary 2.18, we have

Corollary 2.19. If $K$ is an intersection body, then there is the inequality

$$
V(K)^{\frac{n-1}{n}} \leq \frac{\kappa_{n}^{\frac{n-1}{n}}}{\kappa_{n-1}} \max _{u \in S^{n-1}} \operatorname{vol}_{n-1}\left(K \cap u^{\perp}\right)
$$

with equality if and only if $K$ is a ball.

Let $M$ be the cube of volume 1 in Corollary 2.18. Then we obtain for $K \in \mathscr{I}$

$$
V(K)^{\frac{n-1}{n}}<\max _{u \in S^{n-1}} \operatorname{vol}_{n-1}\left(K \cap u^{\perp}\right) .
$$

This shows that $\kappa_{n}^{\frac{n-1}{n}} / \kappa_{n-1}<1$. Corollary 2.19 is related to the maximal slice conjecture. This conjecture states that for $K \in \mathscr{K}_{e}$ there exists a universal constant $c>0$ such that

$$
V(K)^{\frac{n-1}{n}} \leq c \max _{u \in S^{n-1}} \operatorname{vol}_{n-1}\left(K \cap u^{\perp}\right) .
$$

We suspect that $c$ could be 1 . For related problems, see [30].

From Theorem 2.13, we have the following

Theorem 2.20. If a convex body $K \in \mathscr{F}_{e}^{2}$ is not an intersection body, then there exists a convex body $L \in \mathscr{P}$ which is also not an intersection body so that

$$
I_{i} L \subset I_{i} K
$$

but

$$
\tilde{W}_{n-i-1}(K)<\tilde{W}_{n-i-1}(L) .
$$

Proof. Let $M=K$ in (2.14). Since $K$ is not an intersection body, there exists a body $L \in \mathscr{P}$ so that $I_{i} L \subset I_{i} K$, but

$$
\tilde{V}(L, i ; K, 1 ; B)>\tilde{V}(K, i+1 ; B)=\tilde{W}_{n-i-1}(K) .
$$


From (2.8), we have

$$
\begin{aligned}
\tilde{V}(L, i ; K, 1 ; B)^{i+1} & \leq \tilde{V}(L, i+1 ; B)^{i} \tilde{V}(K, i+1 ; B) \\
& =\tilde{W}_{n-i-1}(L)^{i} \tilde{W}_{n-i-1}(K) .
\end{aligned}
$$

Hence,

$$
\tilde{W}_{n-i-1}(K)<\tilde{W}_{n-i-1}(L)
$$

Corollary 2.21. If a body $K \in \mathscr{F}_{e}^{2}$ is not an intersection body, then there exists a body $L \in \mathscr{P}$ which is also not an intersection body so that

$$
I L \subset I K
$$

but

$$
V(K)<V(L)
$$

It should be pointed out that the $C^{\infty}$ case of Corollary 2.21 has been proved by Gardner ([13, Theorem 3.1]), while without convexity it is due to Lutwak ([25, Theorem 12.2]).

Theorem 2.22. The Busemann-Petty problem has an affirmative answer in $\mathbf{R}^{n}$ if and only if each centered convex body in $\mathbf{R}^{n}$ is an intersection body.

Proof. The sufficiency follows from Corollary 2.18. To prove the necessity, it suffices to show that the existence of nonintersection bodies implies a negative answer to the Busemann-Petty problem. Assume that $K$ is not an intersection body. From Theorem 2.13, there exists $L \in \mathscr{F}_{e}^{2}$ so that

$$
I L \subseteq I B \text { and } \tilde{V}_{1}(L, K)>\tilde{V}_{1}(B, K)
$$

Conversely, (2.23) implies that $K$ is not an intersection body. It follows that an infinitesimal deformation of $K$ is not an intersection body either. Therefore, there exist nonintersection bodies in $\mathscr{F}_{e}^{2}$, and Corollary 2.21 gives a negative answer to the Busemann-Petty problem.

The sufficiency in Theorem 2.22 was actually proved by Lutwak (see [25]), which represents the first step towards the full solution of the Busemann-Petty problem. It is proved that no cube in $\mathbf{R}^{n}(n \geq 4)$ is an intersection body [48]. More generally, no polytope in $\mathbf{R}^{n}(n \geq 4)$ is an intersection body [49]. Then the Busemann-Petty problem has a negative answer in $\mathbf{R}^{n}(n \geq 4)$. Gardner [51] has proved that every centered convex body in $\mathbf{R}^{3}$ is an intersection body. This gives a positive answer to the Busemann-Petty problem in $\mathbf{R}^{3}$. For the details of the Busemann-Petty problem, see $[2,3,6,13,14,23,48,51]$.

\section{Characterizations AND INEQUalities of POLAR PROJection bodies}

For a convex body $K$ with the origin in its interior, the polar body $K^{*}$ of $K$ is defined by

$$
K^{*}=\left\{y \in \mathbf{R}^{n}:\langle x, y\rangle \leq 1 \text { for all } x \in K\right\} .
$$

It is easy to verify that $K^{* *}=K$, and for $u \in S^{n-1}$, that

$$
h_{K} \cdot(u)=\rho_{K}(u)^{-1}
$$

while

$$
\rho_{K} \cdot(u)=h_{K}(u)^{-1} \text {. }
$$

Let $\mathscr{Z}^{*}=\left\{K^{*}: K \in \mathscr{Z}\right\}$ which is the set of polar projection bodies. The following lemma is essentially part of a result of Weil ([44, Theorem 4]). 
Lemma 3.1. If $K, L \in \mathscr{K}_{e}$, then $K \subseteq L$ if and only if

$$
V_{1}(M, K) \leq V_{1}(M, L) \text { for all } M \in \mathscr{X}_{e} \text {. }
$$

We recall the centroid body $\Gamma K$ of a star body $K \in \mathscr{S}$ defined by (see [35])

$$
h_{\Gamma K}(x)=\frac{1}{V(K)} \int_{K}|\langle x, y\rangle| d y .
$$

This integral can be written as an integral over $S^{n-1}$ (see, for example, [35])

$$
h_{\Gamma K}(u)=\frac{1}{(n+1) V(K)} \int_{S^{n-1}}|\langle u, v\rangle| \rho_{K}(v)^{n+1} d v
$$

The centroid bodies comprise an important subclass of zonoids. We refer the reader to $[26,29,30]$ for details.

Lemma 3.2. If $K, L \in \mathscr{S}$, then $\Gamma K \subseteq \Gamma L$ if and only if

$$
\tilde{V}_{-1}(K, M) / V(K) \leq \tilde{V}_{-1}(L, M) / V(L), \quad \text { for all } M \in \mathscr{Z}^{*} \text {. }
$$

Proof. For any $M_{0} \in \mathscr{K}_{e}$, let $\Pi^{*} M_{0}=M$. From the identity (see [26, Lemma 9.1])

$$
\tilde{V}_{-1}\left(K, \Pi^{*} L\right) / V(K)=\frac{n+1}{2} V_{1}(L, \Gamma K) \text { for } K \in \mathscr{S}, L \in \mathscr{K},
$$

the inequality (3.3) becomes

$$
V_{1}\left(M_{0}, \Gamma K\right) \leq V_{1}\left(M_{0}, \Gamma L\right), \quad \text { for all } M_{0} \in \mathscr{K}_{e} .
$$

We complete the proof by applying Lemma 3.1.

The necessity part of Lemma 3.2 was proved by Lutwak ([26, Lemma 9.2]). The following lemma is a variant of Theorem 5.1 in [39]. One can give a similar proof to that of Lemma 2.11. For a complete proof see [18].

Lemma 3.5. Let $\mathscr{N}$ be a dense subset of $\mathscr{M}_{e}$. Then a function $h \in C_{e}\left(S^{n-1}\right)$ is the support function of a projection body if and only if

$$
\int_{S^{n-1}} h(u) d \mu(u) \geq 0 \text { whenever } \int_{S^{n-1}}|\langle u, x\rangle| d \mu(x) \geq 0, \mu \in \mathscr{N} .
$$

Lemma 3.6. Let $h \in C_{e}\left(S^{n-1}\right)$. If $h$ is the support function of a projection body, then for all $L, M \in \mathscr{S}$ there is the implication

$$
\Gamma M \subset \Gamma L \Rightarrow\left(h, \rho_{M}^{n+1}\right) / V(M) \leq\left(h, \rho_{L}^{n+1}\right) / V(L) .
$$

Conversely, let $M \in \mathscr{F}_{e}^{2}$ be fixed. If the implication holds for all $L \in \mathscr{P}$, then $h$ is the support function of a projection body.

Proof. From Lemma 3.2, the necessity is clear. To apply Lemma 3.5, we show that the set

$$
\mathscr{N}=\left\{V(L)^{-1} \rho_{L}^{n+1}-c V(M)^{-1} \rho_{M}^{n+1}: c>0, L \in \mathscr{K}_{e}\right\}
$$

is dense in $\mathscr{M}_{e}$. It is enough to show $\mathscr{N}$ is dense in $C_{e}^{2}\left(S^{n-1}\right)$ where the latter is viewed as a subspace of $M_{e}$. Since $M \in \mathscr{F}_{e}^{2}$, for $q \in C_{e}^{2}\left(S^{n-1}\right)$ the function $\rho_{M}+t q$ is the radial function of a body $M_{t} \in \mathscr{F}_{e}^{2}$ for $t$ sufficiently small. We have

$$
V\left(M_{t}\right)^{-1} \rho_{M_{t}}^{n+1}-V(M)^{-1} \rho_{M}^{n+1}=V(M)^{-2} \rho_{M}^{2} T_{M}(q) t+O\left(t^{2}\right)
$$


where

$$
T_{M}(q)=(n+1) V(M) \rho_{M}^{n-2} q-\rho_{M}^{n-1} \int_{S^{n-1}} \rho_{M}^{n-1}(u) q(u) d u, \quad q \in C_{e}^{2}\left(S^{n-1}\right) .
$$

If we can show the set $\mathscr{T}=\left\{T_{M}(q): q \in C_{e}^{2}\left(S^{n-1}\right)\right\}$ is dense in $M_{e}$, then by (3.7) $\mathscr{N}$ is dense in $\mathscr{M}_{e}$. Assume $\mathscr{T}$ is not dense in $\mathscr{M}_{e}$. By the Hahn-Banach theorem, there exists a nontrivial $f \in C_{e}\left(S^{n-1}\right)$ so that

$$
\int_{S^{n-1}} f T_{M}(q) d u=0, \quad \text { for all } q \in C_{e}^{2}\left(S^{n-1}\right) \text {. }
$$

Hence

$$
0=\int_{S^{n-1}} f T_{M}(q) d u=\int_{S^{n-1}} T_{M}(f) q d u
$$

for all $q \in C_{e}^{2}\left(S^{n-1}\right)$. Therefore, $f \in \operatorname{ker} T_{M}$. By integrating both sides of $\rho_{M} T_{M}(f)=0$ over $S^{n-1}$, we obtain

$$
(n+1) V(M) \int_{S^{n-1}} \rho_{M}^{n-1} f d u-n V(M) \int_{S^{n-1}} \rho_{M}^{n-1} f d u=0 .
$$

This gives

$$
\int_{S^{n-1}} \rho_{M}^{n-1} f d u=0
$$

By $T_{M}(f)=0$, we have $f \equiv 0$. This is a contradiction. From Lemma 3.5, we finish the proof of the theorem.

Let $h=\rho_{K}^{-1}$ in Lemma 3.6 and use the formula for the support function of a centroid body. We obtain a characterization of polar projection bodies.

Theorem 3.8. Let $K \in \mathscr{K}_{e}$. If $K$ is a polar projection body, then for all $L, M \in$ $\mathscr{S}$ there is the implication

$$
\Gamma M \subseteq \Gamma L \quad \Longrightarrow \quad \tilde{V}_{-1}(M, K) / V(M) \leq \tilde{V}_{-1}(L, K) / V(L) .
$$

Conversely, let $M \in \mathscr{F}_{e}^{2}$ be fixed. If the implication (3.9) holds for all $L \in \mathscr{P}$, then $K$ is a polar projection body.

Theorem 3.10. Let $K \in \mathscr{K}_{e}$. If $K$ is a polar projection body, then for all $L, M \in$ $\mathscr{S}$

$$
\frac{\tilde{V}_{-1}(L, K)}{\tilde{V}_{-1}(M, K)} \geq \min _{u \in S^{n-1}} \frac{\int_{x \in L}|\langle u, x\rangle| d x}{\int_{x \in M}|\langle u, x\rangle| d x}
$$

Conversely, let $M \in \mathscr{F}_{e}^{2}$ be fixed. If the inequality (3.11) holds for all $L \in \mathscr{P}$, then $K$ is a polar projection body.

Proof. Since (3.11) implies (3.9), the sufficiency is clear. To show the necessity, let $K=\Pi^{*} Q$. Then from (3.4)

$$
\begin{aligned}
\frac{\tilde{V}_{-1}(L, K)}{\tilde{V}_{-1}(M, K)} & =\frac{\tilde{V}_{-1}\left(L, \Pi^{*} Q\right)}{\tilde{V}_{-1}\left(M, \Pi^{*} Q\right)}=\frac{V_{1}(Q, \Gamma L) V(L)}{V_{1}(Q, \Gamma M) V(M)} \\
& =\frac{\int_{S^{n-1}}\left(\int_{x \in L}|\langle u, x\rangle| d x\right) d S_{n-1}(Q ; u)}{\int_{S^{n-1}}\left(\int_{x \in M}|\langle u, x\rangle| d x\right) d S_{n-1}(Q ; u)} \\
& \geq \min _{u \in S^{n-1}} \frac{\int_{x \in L}|\langle u, x\rangle| d x}{\int_{x \in M}|\langle u, x\rangle| d x}
\end{aligned}
$$


Corollary 3.12. Let $K \in \mathscr{Z}^{*}$ and $M \in \mathscr{K}_{e}$. Then

$$
\left[\frac{V(K)}{V(M)}\right]^{\frac{n+1}{n}} \geq \min _{u \in S^{n-1}} \frac{\int_{x \in K}|\langle u, x\rangle| d x}{\int_{x \in M}|\langle u, x\rangle| d x}
$$

with equality if and only if $K$ and $M$ are dilations of each other.

Proof. Note that if $K \in \mathscr{Z}^{*}$ then (3.11) is true for any $M \in \mathscr{X}_{e}$. Let $L=K$ in (3.11). Then

$$
\frac{\tilde{V}_{-1}(L, K)}{\tilde{V}_{-1}(M, K)} \leq \frac{V(K)}{V(M)^{\frac{n+1}{n}} V(K)^{-\frac{1}{n}}}=\left[\frac{V(K)}{V(M)}\right]^{\frac{n+1}{n}}
$$

This gives (3.13).

Corollary 3.14. If $K \in \mathscr{F}_{e}^{2}$ is not a polar projection body, then there exists $L \in \mathscr{P}$ which is also not a polar projection body such that

$$
\Gamma K \subset \Gamma L
$$

but

$$
V(K)>V(L)
$$

Proof. Let $M=K$ in (3.9). Since $K$ is not a polar projection body, there exists a convex body $L \in \mathscr{P}$ so that

$$
\Gamma K \subset \Gamma L
$$

but

$$
V(L)>\tilde{V}_{-1}(L, K) \text {. }
$$

From the dual Minkowski inequality (1.11), we obtain

$$
V(K)>V(L) \text {. }
$$

This corollary was proved by Lutwak ([26, Proposition 9.6]) when $K$ and $L$ are star bodies whose radial functions are $C^{\infty}$.

4. AfFine surface area AND the CURVATURE mMAge OF PROJECTION BOdies

Define

$$
\mathscr{W}=\left\{K \in \mathscr{F}_{e}: f_{K}^{-\frac{1}{n+1}}=h_{Q}, \text { for some } Q \in \mathscr{Z}\right\}
$$

This is an important affine invariant class of convex bodies, called curvature images of projection bodies, see Lutwak [26]. In order to characterize the curvature image of a projection body, we define the affine surface area of $K \in \mathscr{F}$, $A(\boldsymbol{K})$, by

$$
A(K)=\int_{S^{n-1}} f_{K}(u)^{\frac{n}{n+1}} d u .
$$

For $K, L \in \mathscr{F}$, and $i \in \mathbf{R}$, Lutwak [27] defined the $i$ th mixed affine surface area of $K$ and $L, A_{i}(K, L)$, by

$$
A_{i}(K, L)=\int_{S^{n-1}} f_{K}(u)^{\frac{n-i}{n+1}} f_{L}(u)^{\frac{i}{n+1}} d u,
$$

and proved that

$$
A_{-1}(K, L)^{n} \geq A(K)^{n+1} A(L)^{-1}
$$

with equality if and only if $K$ and $L$ are homothetic.

As a consequence of Lemma 3.5, it is easy to show the following (see [19]) 
Lemma 4.4. Let $h \in C_{e}\left(S^{n-1}\right)$. If $h$ is the support function of a projection body, then for all $L, M \in \mathscr{F}_{e}$ there is the implication

$$
\Pi M \subseteq \Pi L \quad \Longrightarrow \quad \int_{S^{n-1}} h(u) d S_{n-1}(M ; u) \leq \int_{S^{n-1}} h(u) d S_{n-1}(L ; u) .
$$

Conversely, let $M \in \mathscr{F}_{e}$ be fixed. If the implication holds for all $L \in \mathscr{P}$, then $h$ is the support function of a projection body.

Let $h=f_{K}^{-\frac{1}{n+1}}$ in Lemma 4.4. It gives the following

Theorem 4.5. Let $K \in \mathscr{F}_{e}$. If $K \in \mathscr{W}$, then for all $L, M \in \mathscr{F}_{e}$ there is the implication

$$
\Pi M \subseteq \Pi L \quad \Longrightarrow \quad A_{-1}(M, K) \leq A_{-1}(L, K) .
$$

Conversely, let $M \in \mathscr{F}_{e}$ be fixed. If the implication holds for all $L \in \mathscr{F}_{e}$, then $K \in \mathscr{W}$.

From Theorem 4.5 and inequality (4.3), we have the following corollary which was proved by Lutwak ([26, Proposition 8.11]) when $K$ has a $C^{\infty}$ curvature function.

Corollary 4.7. If $K \in \mathscr{F}_{e} \backslash \mathscr{W}$, then there exists $L \in \mathscr{F}_{e} \backslash \mathscr{W}$ such that

$$
\Pi K \subset \Pi L
$$

but

$$
A(K)>A(L) .
$$

Proof. Let $M=K$ in Theorem 4.5. If $K \in \mathscr{F}_{e} \backslash \mathscr{W}$, then there exists $L \in \mathscr{F}_{e}$ so that $\Pi K \subset \Pi L$, but

$$
A(K)=A_{-1}(K, K)>A_{-1}(L, K) .
$$

Hence, by (4.3) we get

that is, $A(K)>A(L)$.

$$
A(K)>A_{-1}(L, K) \geq A(L)^{\frac{n+1}{n}} A(K)^{-\frac{1}{n}},
$$

Theorem 4.8. Let $K \in \mathscr{F}_{e}$. If $K \in \mathscr{W}$, then for all $L, M \in \mathscr{F}_{e}$

$$
\frac{A_{-1}(L, K)}{A_{-1}(M, K)} \geq \min _{u \in S^{n-1}} \frac{\operatorname{vol}_{n-1}\left(L \mid u^{\perp}\right)}{\operatorname{vol}_{n-1}\left(M \mid u^{\perp}\right)}
$$

Conversely, let $M \in \mathscr{F}_{e}$ be fixed. If the inequality holds for all $L \in \mathscr{F}_{e}$, then $K \in \mathscr{W}$.

Proof. By (4.6), the condition (4.9) is sufficient to guarantee that $K \in \mathscr{W}$. Conversely, let $f_{K}^{-\frac{1}{n+1}}=h_{Q}$ for some $Q \in \mathscr{Z}$. Then

$$
\frac{A_{-1}(L, K)}{A_{-1}(M, K)}=\frac{\left(f_{L}, h_{Q}\right)}{\left(f_{M}, h_{Q}\right)} \text {. }
$$

Since every centered zonoid is a projection body, let $Q=\Pi Q_{0}$ for some $Q_{0} \in$ $\mathscr{K}_{e}$. We have

$$
\begin{aligned}
\frac{\left(f_{L}, h_{Q}\right)}{\left(f_{M}, h_{Q}\right)} & =\frac{V_{1}\left(L, \Pi Q_{0}\right)}{V_{1}\left(M, \Pi Q_{0}\right)}=\frac{V_{1}\left(Q_{0}, \Pi L\right)}{V_{1}\left(Q_{0}, \Pi M\right)} \\
& =\frac{\int_{S^{n-1}} \operatorname{vol}_{n-1}\left(L \mid u^{\perp}\right) d S_{n-1}\left(Q_{0} ; u\right)}{\int_{S^{n-1}} \operatorname{vol}_{n-1}\left(M \mid u^{\perp}\right) d S_{n-1}\left(Q_{0} ; u\right)} \geq \min _{u \in S^{n-1}} \frac{\operatorname{vol}_{n-1}\left(L \mid u^{\perp}\right)}{\operatorname{vol}_{n-1}\left(M \mid u^{\perp}\right)} .
\end{aligned}
$$

This proves the theorem. 
Corollary 4.10. If $K \in \mathscr{W}$ and $M \in \mathscr{F}_{e}$, then

$$
\left[\frac{A(K)}{A(M)}\right]^{\frac{n+1}{n}} \geq \min _{u \in S^{n-1}} \frac{\operatorname{vol}_{n-1}\left(K \mid u^{\perp}\right)}{\operatorname{vol}_{n-1}\left(M \mid u^{\perp}\right)}
$$

with equality if and only if $K$ and $M$ are homothetic.

Proof. By (4.3) we have

$$
\frac{A_{-1}(L, K)}{A_{-1}(M, K)} \leq \frac{A(K)}{A(M)^{\frac{a+1}{n}} A(K)^{-\frac{1}{n}}}=\left[\frac{A(K)}{A(M)}\right]^{\frac{n+1}{n}} .
$$

This proves the corollary.

The above corollary implies that for $K \in \mathscr{W}$ and $M \in \mathscr{F}_{e}$ if

$$
\operatorname{vol}_{n-1}\left(K \mid u^{\perp}\right) \geq \operatorname{vol}_{n-1}\left(M \mid u^{\perp}\right) \text { for all } u \in S^{n-1} \text {, }
$$

then

$$
A(K) \geq A(M) .
$$

This result is due to Lutwak ([26, Theorem 8.9]).

Corollary 4.11. If $K \in \mathscr{W}$, then

$$
A(K)^{\frac{n+1}{n}} \geq \frac{\left(n \kappa_{n}\right)^{\frac{n+1}{n}}}{\kappa_{n-1}} \min _{u \in S^{n-1}} \operatorname{vol}_{n-1}\left(K \mid u^{\perp}\right)
$$

with equality if and only if $K$ is a ball.

For affine inequalities about the projection body and the affine surface area, see Lutwak [29].

\section{GENERALIZED INTERSECTION BODIES}

For $f \in C\left(S^{n-1}\right)$, the cosine transform of $f, C f$, is defined on $S^{n-1}$ by

$$
(C f)(u)=\frac{1}{2} \int_{S^{n-1}}|\langle u, v\rangle| f(v) d v \quad \text { for } u \in S^{n-1} .
$$

By Fubini's theorem, the cosine transform is selfadjoint, i.e., for $f, g \in C\left(S^{n-1}\right)$,

$$
(f, C g)=(C f, g) \text {. }
$$

When restricted to $C_{e}^{\infty}\left(S^{n-1}\right)$, the cosine transform,

$$
C: C_{e}^{\infty}\left(S^{n-1}\right) \longrightarrow C_{e}^{\infty}\left(S^{n-1}\right),
$$

is a continuous bijection (see Schneider [38]). Then the cosine transform $C$ is a continuous bijection of $\mathscr{D}_{e}\left(S^{n-1}\right)$ to itself, when $\mathscr{D}_{e}\left(S^{n-1}\right)$ is given the strong topology (see Goodey and Weil $\left[17\right.$, p. 677]). For $K \in \mathscr{H}_{e}$, the distribution $C^{-1} h_{K}$ is called the generating distribution of $K$, and denoted by $\mu_{K}$. From (1.7), a convex body $K \in \mathscr{K}_{e}$ is a zonoid if and only if $\mu_{K}$ is a measure, and is a generalized zonoid if and only if $\mu_{K}$ is a signed measure. By using spherical harmonics, Schneider [38] proved that if $f \in C_{e}^{k}\left(S^{n-1}\right)(k=n+2$ when $n$ is even and $k=n+3$ when $n$ is odd), then there exists $g \in C_{e}\left(S^{n-1}\right)$ such that $C g=f$. This implies that the class of generalized zonoids is dense in the class of centered convex bodies (see [38]). Schneider's techniques have inspired 
many works, in particular, on projection bodies and intersection bodies (see $[17,25])$.

By using the techniques for projection bodies in [17], we apply the $L^{p}$ estimates for the spherical Radon transform to show that every centered body in $\mathbf{R}^{n}$ of class $C^{k}, k=\left[\frac{n+1}{2}\right]-1$, is a generalized intersection body. This implies that the class of generalized intersection bodies is dense in the class of centered bodies. Since $S^{n-1}$ is compact, every distribution on $S^{n-1}$ is of finite order. It turns out that the dual generating distribution has order at most $\left[\frac{n+1}{2}\right]-1$. For dual mixed volume characterizations of generalized intersection bodies, see [18]. The approach of this section using the $L^{p}$ estimates of the spherical Radon transform and the Sobolev embedding theorem was suggested by E. Grinberg.

Let $L^{2}\left(S^{n-1}\right)$ be the space of even $L^{2}$-functions on $S^{n-1}$. Denote by $L_{s}^{2}\left(S^{n-1}\right), s \geq 0$, the Sobolev spaces on $S^{n-1}$. The spherical harmonic expansion $\sum_{i=0}^{\infty} f_{i}$ of $f \in L_{s}^{2}\left(S^{n-1}\right)$ satisfies (see [43, (4.5)])

$$
\|f\|_{L_{s}}^{2}=\sum_{i=0}^{\infty}\left(1+i^{2}\right)^{s}\left\|f_{i}\right\|_{2}^{2}<\infty
$$

For $s<0$, the Sobolev space $L_{s}^{2}\left(S^{n-1}\right)$ is defined as the dual space of $L_{-s}^{2}\left(S^{n-1}\right)$. Strichartz [43] proved that the spherical Radon transform

$$
R: L_{s}^{2}\left(S^{n-1}\right) \longrightarrow L_{s+\frac{n-2}{2}}^{2}\left(S^{n-1}\right)
$$

is a bijection. He also proved that there is a constant $b$ such that

$$
b^{-1}\|R f\|_{L_{s+\frac{n-2}{2}}} \leq\|f\|_{L_{s}} \leq b\|R f\|_{L_{s+\frac{n-2}{2}}} .
$$

Since $S^{n-1}$ is compact, there is the inclusion

$$
C_{e}^{k}\left(S^{n-1}\right) \subset L_{k}^{2}\left(S^{n-1}\right) \text { for } k \geq 0 .
$$

From (5.1) and (5.3), we can find conditions which force the dual generating distribution to be an $L^{2}$ function.

Theorem 5.4. If $K \in \mathscr{S}_{e}$ has the radial function $\rho_{K} \in C_{e}^{k}\left(S^{n-1}\right), k=\left[\frac{n+1}{2}\right]-1$, then $K$ is a generalized intersection body.

Corollary 5.5. The generalized intersection bodies are dense in the class of centered bodies.

Goodey and Weil [17] showed that for a centered convex body $K$ the generating distribution $\mu_{K}$ has degree at most $\frac{n+5}{2}$. For the dual generating distribution (see $\S 2$ ) we have the following result.

Theorem 5.6. If $K$ is a centered body in $\mathbf{R}^{n}$, then the dual generating distribution $\tilde{\mu}_{K}=R^{-1} \rho_{K}$ is a generalized function of class $L_{\frac{2-n}{2}}^{2}\left(S^{n-1}\right)$, and hence it has order at most $\left[\frac{n+1}{2}\right]-1$.

Proof. Since (5.1) is a bijection, for $f \in L_{\frac{n-2}{2}}^{2}\left(S^{n-1}\right)$ there is $g \in L^{2}\left(S^{n-1}\right)$ so that $f=R g$. From the Hölder inequality and (5.2), we have

$$
\left|\left(\rho_{K}, R^{-1} f\right)\right| \leq\left\|\rho_{K}\right\|_{2}\|g\|_{2} \leq b\left\|\rho_{K}\right\|_{2}\|f\|_{L_{\frac{n-2}{2}}} .
$$


We extend $\tilde{\mu}_{K}$ to the linear functional on $L_{\frac{n-2}{2}}^{2}\left(S^{n-1}\right)$ defined by

$$
f \rightarrow\left(\rho_{K}, R^{-1} f\right) \text {. }
$$

Then $\tilde{\mu}_{K}$ is a continuous functional on $L_{\frac{n-2}{2}}^{2}\left(S^{n-1}\right)$, i.e., $\tilde{\mu}_{K} \in L_{\frac{2-n}{2}}^{2}\left(S^{n-1}\right)$. Let $k=\left[\frac{n+1}{2}\right]-1$. Then $k \geq \frac{n-2}{2}$. From (5.3), we have the inclusion of dual spaces

$$
C_{e}^{k}\left(S^{n-1}\right)^{*} \supset L_{k}^{2}\left(S^{n-1}\right)^{*}=L_{-k}^{2}\left(S^{n-1}\right) .
$$

Hence,

$$
\tilde{\mu}_{K} \in C_{e}^{k}\left(S^{n-1}\right)^{*},
$$

that is, $\tilde{\mu}_{K}$ has order at most $k$.

Lemma 5.7. If $f \in C_{e}^{n+k-1}\left(S^{n-1}\right)$, then there exists $g \in C_{e}^{k}\left(S^{n-1}\right)$ such that $R g=f$.

Proof. By the Sobolev embedding theorem (see $[1$, p. 35]), we have

$$
L_{s}^{2}\left(S^{n-1}\right) \subset C^{k}\left(S^{n-1}\right), \quad \text { for } s>k+\frac{n-1}{2} .
$$

Since (5.1) is a bijection, it suffices to show the inclusion

$$
C_{e}^{n+k-1}\left(S^{n-1}\right) \subset L_{s+\frac{n-2}{2}}^{2}\left(S^{n-1}\right)
$$

for some $s>k+\frac{n-1}{2}$. From (5.3) one can choose $s=k+\frac{n}{2}$.

The above lemma implies the following

Theorem 5.8. If a centered body $K$ such that $\rho_{K} \in C_{e}^{n-1}\left(S^{n-1}\right)$ is an intersection body, then $K$ is the intersection body of another centered body.

Let $\Delta$ be the Laplacian on $S^{n-1}$. By using the relation $(n-1) R=$ $(\Delta+n-1) C$ (see Goodey and Weil [17]) and the Remark 6.7(i) (the operator $L_{M, 1}=\Delta+n-1$ is independent of $M$ ), we conclude the following results: If $f \in C_{e}^{k+n+1}\left(S^{n-1}\right)$, then there exists $g \in C_{e}^{k}\left(S^{n-1}\right)$ such that $C g=f$.

\section{Curvature functions}

In this section we discuss a type of selfadjoint elliptic differential operator associated with a convex body. These operators are applied to show the openness of the class of curvature functions with Hölder continuity. A consequence of the openness is a strengthening of Weil's theorem in [47] about the denseness of the difference of $j$ th surface area measures in $M_{e}$. Finally, we prove an existence theorem about deformations of convex hypersurfaces, which is a companion to a well-known uniqueness theorem in global differential geometry.

If $f_{j}(M ; \cdot)$ is the $j$ th elementary symmetric function of radii of principal curvature of $M \in \mathscr{F}_{e}^{2}$, then $S_{j}(M ; \cdot)=f_{j}(M ; \cdot) S_{n-1}(B ; \cdot)$. For $g \in$ $C_{e}^{2}\left(S^{n-1}\right)$ let $M(t)$ be the convex body with support function $h_{M}+t g$. We consider the operator

$$
L_{M, j}(g)=\left.\frac{d}{d t}\right|_{t=0} f_{j}(M(t) ; \cdot), \quad g \in C_{e}^{2}\left(S^{n-1}\right) .
$$


Let $\left\{e_{1}, \cdots, e_{n-1}\right\}$ be an orthonormal frame on $S^{n-1}$. Let $\left(h_{i k}\right)$ be the Hessian matrix of $h_{M}$ with respect to the frame. Then the eigenvalues of the matrix $\left(h_{i k}+h_{M} \delta_{i k}\right)$ are the radii of curvature of $M$ (see, for example, [20, Part IV, 32, p. 37]). Let $c_{i k}=h_{i k}+h_{M} \delta_{i k}$. The radii of principal curvature of $M(t)$ are the eigenvalues of the matrix $\left(c_{i k}(t)\right)=\left(h_{i k}+h_{M} \delta_{i k}+t g_{i k}+t g \delta_{i k}\right)$, where $\left(g_{i k}\right)$ is the Hessian of $g$. Hence

$$
\begin{aligned}
\left.\frac{d}{d t}\right|_{t=0} f_{j}(M(t) ; \cdot) & =\left.\sum_{i, k=1}^{n-1} \frac{\partial f_{j}(M(t) ; \cdot)}{\partial c_{i k}(t)} \frac{d c_{i k}(t)}{d t}\right|_{t=0} \\
& =\sum_{i, k=1}^{n-1} \frac{\partial f_{j}(M ; \cdot)}{\partial c_{i k}}\left(g_{i k}+g \delta_{i j}\right),
\end{aligned}
$$

and we obtain

$$
L_{M, j}(g)=\sum_{i, k=1}^{n-1} \frac{\partial f_{j}(M ; \cdot)}{\partial c_{i k}} g_{i k}+(n-j) f_{j-1}(M ; \cdot) g .
$$

Therefore, the operator $L_{M, j}$ is a linear differential operator of second order. Diagonalize $c_{i k}$ so that $c_{i k}=r_{i} \delta_{i k}$, where $r_{i}$ are the radii of curvature of $M$. Then the matrix

$$
\left(\frac{\partial f_{j}(M ; \cdot)}{\partial c_{i k}}\right)=\left(\frac{\partial f_{j}(M ; \cdot)}{\partial r_{i}} \delta_{i k}\right)
$$

is positive definite, that is, $L_{M, j}$ is elliptic.

For $g \in C_{e}^{2}\left(S^{n-1}\right)$ there exist $K, L \in \mathscr{X}_{e}$ so that $g=h_{K}-h_{L}$. By using (1.3), we have

$$
\begin{aligned}
& \left.\frac{d}{d t}\right|_{t=0} S_{j}(M(t) ; \cdot) \\
& =j[S(K, \underbrace{M, \cdots, M}_{j-1}, \underbrace{B, \cdots, B}_{n-j-1} ; \cdot)-S(L, \underbrace{M, \cdots, M}_{j-1}, \underbrace{B, \cdots, B}_{n-j-1} ; \cdot)] .
\end{aligned}
$$

Hence

$$
\begin{aligned}
& L_{M, j}(g) S_{n-1}(B ; \cdot) \\
& =j[S(K, \underbrace{M, \cdots, M}_{j-1}, \underbrace{B, \cdots, B}_{n-j-1} ; \cdot)-S(L, \underbrace{M, \cdots, M}_{j-1}, \underbrace{B, \cdots, B}_{n-j-1} ; \cdot)] .
\end{aligned}
$$

This gives

$$
\int_{S^{n-1}} g(u) L_{M, j}(f)(u) d S_{n-1}(B ; u)=\int_{S^{n-1}} f(u) L_{M, j}(g)(u) d S_{n-1}(B ; u)=0
$$

for all $g, f \in C_{e}^{2}\left(S^{n-1}\right)$. This shows that $L_{M, j}$ is formally selfadjoint. Note that for $j=1$ we have $L_{M, 1}=\Delta+n-1$, where $\Delta$ is the Laplacian on $S^{n-1}$.

Lemma 6.1. If $M \in \mathscr{F}_{e}^{2, \alpha}$, then the operator

$$
L_{M, j}: C_{e}^{2, \alpha}\left(S^{n-1}\right) \rightarrow C_{e}^{\alpha}\left(S^{n-1}\right)
$$

is bijective.

Proof. The injectivity of the operator $L_{M, j}$ follows from the equality conditions of the Alexandrov-Fenchel inequality (see [52, p. 359; 19]). To prove 
the surjectivity, first assume $h_{M} \in C_{e}^{\infty}\left(S^{n-1}\right)$. Then $L_{M, j}$ is an elliptic and formally selfadjoint operator with $C^{\infty}$ coefficients. We have (see, for example, $[50$, p. 258])

$$
C^{\infty}\left(S^{n-1}\right)=\operatorname{im} L_{M, j} \oplus \operatorname{ker} L_{M, j} .
$$

Hence, $L_{M, j}$ is surjective. Also, one can use the argument of Cheng and Yau $([12$, p. 508$])$ to show

$$
L_{M, j}: C_{e}^{k+2, \alpha}\left(S^{n-1}\right) \longrightarrow C_{e}^{k, \alpha}\left(S^{n-1}\right)
$$

is surjective for $k$ sufficiently large.

If $h_{M} \in C_{e}^{2, \alpha}\left(S^{n-1}\right)$, then $L_{M, j}$ has $C^{\alpha}$-coefficients. We can write $L_{M}$ as

$$
L_{M, j}=\sum a_{i k} \partial_{i k}+\sum b_{i} \partial_{i}+c
$$

where $\partial_{i}, \partial_{i k}$ are the covariant derivatives on the sphere $S^{n-1}$ and $c$ is a function on $S^{n-1}$. Choose positive number $\sigma>0$ so that $c-\sigma<0$. Consider the equation

$$
\left(L_{M, j}-\sigma\right) g=f, \quad f \in C_{e}^{\alpha}\left(S^{n-1}\right) .
$$

Approximate the coefficients of this equation by $C^{\infty}$-coefficients,

$$
\left(L_{M_{\nu}, j}-\sigma\right) g=\sum a_{i k}^{\nu} \partial_{i k} g+\sum b_{i}^{\nu} \partial_{i} g+\left(c^{\nu}-\sigma\right) g=f_{\nu}, \quad \nu=1,2, \cdots,
$$

where $h_{M_{\nu}} \in C_{e}^{\infty}\left(S^{n-1}\right)$ and $h_{M_{\nu}} \rightarrow h_{M}$ uniformly in $C_{e}^{2, \alpha}\left(S^{n-1}\right)$. Each equation has a unique solution $g_{\nu} \in C_{e}^{\infty}\left(S^{n-1}\right)$. We show that the solutions $\left\{g_{\nu}\right\}$ are uniformly bounded in $C^{2, \alpha}\left(S^{n-1}\right)$. Since $c^{\nu} \rightarrow c$ and $c-\sigma<0$, there exists a constant $c_{0}<0$ so that $c^{\nu}-\sigma<c_{0}$. Assume that $g_{\nu}$ attains its maximum at $x_{1}$. Then the Hessian $\left(\partial_{i k} g_{\nu}\left(x_{1}\right)\right)$ is seminegative. Hence, $\sum a_{i k}^{\nu} \partial_{i k} g_{\nu}\left(x_{1}\right) \leq 0$. If $g_{\nu}\left(x_{1}\right)>0$, then

$$
\operatorname{cog}_{\nu}\left(x_{1}\right) \geq\left(c^{\nu}-\sigma\right) g_{\nu}\left(x_{1}\right) \geq f_{\nu}\left(x_{1}\right) \text {. }
$$

Hence

$$
g_{\nu}\left(x_{1}\right) \leq\left|c_{0}\right|^{-1}\left|f_{\nu}\left(x_{1}\right)\right| .
$$

This is obviously true if $g_{\nu}\left(x_{1}\right) \leq 0$. Assume that $g_{\nu}$ attains its minimum at $x_{0}$. Then the Hessian of $g_{\nu}$ at $x_{0}$ is semipositive. If $g_{\nu}\left(x_{0}\right)<0$, then

$$
c_{0} g_{\nu}\left(x_{0}\right) \leq\left(c^{\nu}-\sigma\right) g_{\nu}\left(x_{0}\right) \leq f_{\nu}\left(x_{0}\right) \text {. }
$$

Hence

$$
-g_{\nu}\left(x_{0}\right) \leq\left|c_{0}\right|^{-1}\left|f_{\nu}\left(x_{0}\right)\right| .
$$

Also, this is true if $g_{\nu}\left(x_{0}\right) \geq 0$. Therefore, (6.4) and (6.5) give that

$$
\left\|g_{\nu}\right\|_{C^{0}} \leq\left|c_{0}\right|^{-1}\left\|f_{\nu}\right\|_{C^{0}} \text {. }
$$

By Schauder interior estimates (see $[1$, p. $88 ; 15, \S 6.1]$ ) and the compactness of $S^{n-1}$, the sequence $\left\{g_{\nu}\right\}$ is uniformly bounded in $C^{2, \alpha}\left(S^{n-1}\right)$. Hence, the functions $g_{\nu}$ and their first and second derivatives are equicontinuous. From the Ascoli-Arzelà theorem, there is a convergent subsequence in $C^{2}\left(S^{n-1}\right)$. Hence the equation (6.3) has a solution $u \in C_{e}^{2, \alpha}\left(S^{n-1}\right)$. Therefore, the operator

$$
L_{M, j}-\sigma: C_{e}^{2, \alpha}\left(S^{n-1}\right) \longrightarrow C_{e}^{\alpha}\left(S^{n-1}\right)
$$


is surjective. In view of the maximum principle (see $[1$, p. 96; 15, §3.2]), it is easy to see that this operator is also injective. Note that above argument implies that the inverse operator

$$
\left(L_{M, j}-\sigma\right)^{-1}: C_{e}^{\alpha}\left(S^{n-1}\right) \longrightarrow C_{e}^{2, \alpha}\left(S^{n-1}\right)
$$

sends every uniformly bounded sequence of functions in $C_{e}^{\alpha}\left(S^{n-1}\right)$ to a sequence of functions in $C_{e}^{2, \alpha}\left(S^{n-1}\right)$ which has a uniformly convergent subsequence, that is, $\left(L_{M, j}-\sigma\right)^{-1}$ is compact. Therefore, the inverse operator

$$
\left(L_{M, j}-\sigma\right)^{-1}: C_{e}^{\alpha}\left(S^{n-1}\right) \longrightarrow C_{e}^{\alpha}\left(S^{n-1}\right)
$$

is also compact. Consider the equation

$$
g+\sigma\left(L_{M, j}-\sigma\right)^{-1} g=\left(L_{M, j}-\sigma\right)^{-1} f, \quad f \in C_{e}^{\alpha}\left(S^{n-1}\right) .
$$

By applying Fredholm alternates to $\left(L_{M, j}-\sigma\right)^{-1}$ on the Banach space $C_{e}^{\alpha}\left(S^{n-1}\right)$, we conclude that (6.6) has a solution $g \in C_{e}^{\alpha}\left(S^{n-1}\right)$ because the homogeneous equation has only trivial solution. Since $\left(L_{M, j}-\sigma\right)^{-1} g,\left(L_{M, j}-\sigma\right)^{-1} f \in$ $C_{e}^{2, \alpha}\left(S^{n-1}\right)$, we conclude that the equation

$$
L_{M, j} g=f, \quad f \in C_{e}^{\alpha}\left(S^{n-1}\right),
$$

has a solution $g \in C_{e}^{2, \alpha}\left(S^{n-1}\right)$. Therefore, (6.2) is surjective.

Remark 6.7. (i) By regularity theorems (see $[1$, p. 85]), the operator

$$
L_{M, j}: C_{e}^{k+2, \alpha}\left(S^{n-1}\right) \longrightarrow C_{e}^{k, \alpha}\left(S^{n-1}\right)
$$

is bijective if $M \in \mathscr{F}_{e}^{k+2, \alpha}$ for $k \geq 0$. From the proof of Lemma 6.1 one can see that if one drops the symmetry assumption on $M$ then Lemma 6.1 takes the form

where

$$
C^{\alpha}\left(S^{n-1}\right)=\operatorname{im} L_{M, j} \oplus \operatorname{ker} L_{M, j},
$$

$$
L_{M, j}: C^{2, \alpha}\left(S^{n-1}\right) \longrightarrow C^{\alpha}\left(S^{n-1}\right), \quad M \in \mathscr{F}^{2, \alpha},
$$

and $\operatorname{ker} L_{M, j}$ consists of first order spherical harmonic functions.

(ii) In two dimensions Lemma 6.1 is due to D. Hilbert and is related to the Minkowski problem (see Nirenberg [31, §15]). The ellipticity, selfadjointness and injectivity of $L_{M, j}$ are well-known (see $\left.[32,34]\right)$. The author has not found a proof for the surjectivity of $L_{M, j}$ in the literature. The above proof is apparently not straightforward. All these properties of $L_{M, j}$ are used by Pogorelov in his work on the Minkowski problem (see [37, pp. 46-49 and 6061]).

The bijectivity of the operator $L_{M, j}$ can be applied to show the openness of the class of curvature functions $f_{j}(M ; \cdot)$ with Hölder continuity, and to prove existence and uniqueness theorems about the deformation of $M \in \mathscr{F}_{e}^{2, \alpha}$ which preserves normals.

Theorem 6.8. Let $f_{j}$ be the $j$ th curvature function of a convex body $M \in \mathscr{F}_{e}^{2, \alpha}$. Then there exists a neighborhood $U$ of $f_{j}$ in $C_{e}^{\alpha}\left(S^{n-1}\right)$ such that every function in $U$ is the jth curvature function of a convex body in $\mathscr{F}_{e}^{2, \alpha}$.

Proof. Define an operator

$$
F_{j}: C^{2, \alpha}\left(S^{n-1}\right) \longrightarrow C^{\alpha}\left(S^{n-1}\right)
$$


Let $\left(h_{i k}\right)$ be the Hessian matrix of $h \in C^{2, \alpha}\left(S^{n-1}\right)$ with respect to a normal frame on $S^{n-1}$. Then $F_{j}(h)$ is defined as the sum of all $j$-rowed principal minors of the matrix $\left(h_{i k}+h \delta_{i k}\right)$. If $h$ is the support function of a body $M \in \mathscr{F}_{e}^{2, \alpha}$, then $F_{j}(h)$ is the $j$ th curvature function $f_{j}(M ; \cdot)$ of $M$. We want to show that for a function $f$ in a neighborhood of $f_{j}(M ; \cdot)$ in $C_{e}^{\alpha}\left(S^{n-1}\right)$, one can always solve the equation $F_{j}(h)=f$, where $h$ is the support function of a body in $\mathscr{F}_{e}^{2, \alpha}$. As is well known (see [41]), it suffices to show that the linearized operator $L_{M, j}$ of $F_{j}$ at $h_{M}$ is surjective. From Lemma 6.1, we conclude Theorem 6.8.

If $j=n-1$ and $M \in \mathscr{F}_{e}^{5}$, Theorem 6.8 has proved by Cheng and Yau [12, $\S 4]$, while the case of $n=3$ is due to Nirenberg [31, \$15].

Corollary 6.9. Every function in $C_{e}^{\alpha}\left(S^{n-1}\right)$ is a difference of $j$ th curvature functions of convex bodies in $\mathscr{F}_{e}^{2, \alpha}$.

Proof. For $f \in C_{e}^{\alpha}\left(S^{n-1}\right)$ and $M \in \mathscr{F}_{e}^{2, \alpha}, f_{j}(M ; \cdot)+t f$ converges to $f_{j}(M ; \cdot)$ in $C_{e}^{\alpha}\left(S^{n-1}\right)$ when $t \rightarrow 0$. By Theorem 6.8, there exists $M(t) \in \mathscr{F}_{e}^{2, \alpha}$ so that $f_{j}(M(t) ; \cdot)=f_{j}(M ; \cdot)+t f$ when $t$ is sufficiently small. Hence

$$
f=f_{j}\left(t^{-\frac{1}{j}} M(t) ; \cdot\right)-f_{j}\left(t^{\frac{1}{j}} M ; \cdot\right) .
$$

The formula (6.10) gives the following corollary, which is a strengthening of Weil's Theorem 3.5 in [46].

Corollary 6.11. Let $M \in \mathscr{F}_{e}^{2, \alpha}$ and $j \in\{1, \cdots, n-1\}$. Then the set

$$
\left\{S_{j}(L ; \cdot)-c S_{j}(M ; \cdot): c>0, L \in \mathscr{F}_{e}^{2, \alpha}\right\}
$$

is dense in $M_{e}$.

We turn to the deformation of hypersurfaces. Let $X$ be a hypersurface of class $C^{2}$ in $\mathbf{R}^{n}$, and let $l: X \longrightarrow \mathbf{R}^{n}$ be the isometric embedding. A deformation of $X$ is a 1-parameter family of hypersurfaces $X(t)$ which is defined by

$$
l(t)=\imath+t Z, \quad-\varepsilon<t<\varepsilon,
$$

where $Z: X \rightarrow \mathbf{R}^{n}$ is of class $C^{2}$. For $M \in \mathscr{F}^{2}$, the boundary $\partial M$ is a hypersurface of class $C^{2}$ which has positive Gaussian curvature. At each point $x \in \partial M$ there exists a unique outer normal vector $u \in S^{n-1}$. This gives a diffeomorphism from $\partial M$ to $S^{n-1}$, called the Gauss map of $\partial M$. We can parameterize $x(u) \in \partial M$ via the Gauss map, which is called the normal parameterization of $\partial M$. Then the deformation (6.12) of $\partial M$ can be written as

$$
x(u, t)=x(u)+t Z(u), \quad-\varepsilon<t<\varepsilon .
$$

It defines a family of convex bodies $M(t) \in \mathscr{F}^{2}$. A deformation is called symmetric if $Z(u)$ is even. If $x(u, t)$ is the normal parameterization of $\partial M(t)$, the deformation (6.13) is said to preserve normals. Denote by $\delta f_{j}$ the variation of the $j$ th curvature function of $M$. If a deformation of $\partial M$ preserves normals and satisfies $\delta f_{j}=0$, then the deformation is trivial, i.e., $\partial M(t)$ is a translation of $\partial M$. This is a well-known uniqueness theorem in global differential geometry. For references and generalizations, see [33,34]. We prove the related existence theorem. 
Theorem 6.14. Let $f_{j}$ be the $j$ th curvature function of a convex body $M \in$ $\mathscr{F}_{e}^{2, \alpha}$. Then for any $f \in C_{e}^{\alpha}\left(S^{n-1}\right)$ there exists a unique symmetric deformation of $M$ which preserves normals such that $\delta f_{j}=f$.

Proof. Let $g$ be the unique solution to the equation $L_{M, j}(g)=f$. Construct a family of convex bodies $M_{1}(t)$ with support function $h_{M}+t g$. Obviously, this family of convex bodies gives a symmetric deformation of $M$ which preserves normals. We have

$$
\delta f_{j}=\left.\frac{d}{d t}\right|_{t=0} f_{j}(M(t) ; \cdot)=L_{M, j}(g)=f .
$$

Let $x(u) \in \partial M$ be the normal parameterization. Let $M(t)$,

$$
x(u, t)=x(u)+t Z(u) \in \partial M(t), \quad-\varepsilon<t<\varepsilon,
$$

be any symmetric deformation of $M$ which preserves normals and satisfies $\delta f_{j}=f$. Then the support function of $M(t)$ is $h_{M}+t\langle Z, u\rangle$. Hence,

$$
L_{M, j}(\langle Z, u\rangle)=f .
$$

This implies $\langle Z, u\rangle=g$. Consequently, $M(t)$ and $M_{1}(t)$ have the same support function, that is, they are congruent.

\section{REFERENCES}

1. T. Aubin, Nonlinear analysis on manifolds. Monge-Ampère equations, Springer-Verlag, 1982.

2. K. Ball, Some remarks on the geometry of convex sets, Geometric Aspects of Functional Analysis, 1986-1987, Lecture Notes in Math., vol. 1317, Springer, 1988.

3. M. Berger, Convexity, Amer. Math. Monthly 97 (1990), 650-678.

4. E. D. Bolker, A class of convex bodies, Trans. Amer. Math. Soc. 145 (1969), 323-345.

5. T. Bonnesen and W. Fenchel, Theorie der konvexen Körper, Springer-Verlag, Berlin, 1934.

6. J. Bourgain, On the Busemann-Petty problem for perturbations of ball, Geom. Funct. Anal. 1 (1991), 1-13.

7. Yu. D. Burago and V. A. Zalgaller, Geometric inequalities, Springer-Verlag, Berlin, 1988.

8. H. Busemann, $A$ theorem on convex bodies of the Brunn-Minkowski type, Proc. Nat. Acad. Sci. U.S.A. 35 (1949), 27-31.

9. H. Busemann and C. M. Petty, Problems on convex bodies, Math. Scand. 4 (1956), 88-94.

10. H. Busemann, Convex surfaces, Interscience, New York, 1958.

11. G. D. Chakerian, Sets of constant relative width and constant relative brightness, Trans. Amer. Math. Soc. 129 (1967), 26-37.

12. S. Y. Cheng and S. T. Yau, On the regularity of the solution of the n-dimensional Minkowski problem, Comm. Pure Appl. Math. 29 (1976), 495-516.

13. R. Gardner, Intersection bodies and the Busemann-Petty problem, Trans. Amer. Math. Soc. 342 (1994), 435-445.

14. A. Giannopoulos, A note on a problem of $H$. Busemann and C. M. Petty concerning sections of symmetric convex bodies, Mathematika 37 (1990), 239-244.

15. D. Gilbarg and N. Trudinger, Elliptic partial differential equations of second order, SpringerVerlag (1977).

16. P. R. Goodey, Centrally symmetric convex sets and mixed volumes, Mathematika 24 (1977), 193-198.

17. P. Goodey and W. Weil, Centrally symmetric convex bodies and the spherical Radon transform, J. Differential Geometry 35 (1992), 675-688. 
18. P. Goodey, E. Lutwak and W. Weil, Functional analytic characterizations of classes of convex bodies, (to appear).

19. P. Goodey and G. Zhang, Characterizations and inequalities of zonoids, (to appear).

20. E. L. Grinberg and I. Rivin, Infinitesimal aspects of the Busemann and Petty problem, Bull. London Math. Soc. 22 (1990), 478-484.

21. P. Hartman and A. Wintner, On the third fundamental form of a surface, Amer. J. Math. 75 (1953), 298-334.

22. S. Helgason, Groups and geometric analysis, Academic Press, 1984.

23. D. G. Larman and C. A. Rogers, The existence of a centrally symmetric convex body with central cross-sections that are unexpectedly small, Mathematika 22 (1975), 164-175.

24. E. Lutwak, Volume of mixed bodies, Trans. Amer. Math. Soc. 294 (1986), 487-500.

25. Intersection bodies and dual mixed volumes, Adv. Math. 71 (1988), 232-261.

26. __ Centroid bodies and dual mixed volumes, Proc. London Math. Soc. (3) 60 (1990), 365-391.

27. __ Mixed affine surface area, J. Math. Anal. Appl. 125 (1987), 351-360.

28. __ Dual mixed volumes, Pacific J. Math. 58 (1975), 531-538.

29. __ Selected affine isoperimetric inequalities, Convex Geometry (P. M. Gruber and J. M. Wills, eds.), Elsevier Science Publishers, 1993.

30. V. D. Milman and A. Pajor, Isotropic position and inertia ellipsoids and zonoids of the unit ball of a normed n-dimensional space, Geometric Aspects of Functional Analysis (J. Lindenstrauss and V. D. Milman, eds.), Lecture Notes in Math., vol. 1376, Springer-Veriag, Berlin, 1989, pp. 64-104.

31. L. Nirenberg, The Weyl and Minkowski problems in differential geometry in the large, Comm. Pure Appl. Math. 6 (1953), 337-394.

32. V. I. Oliker, On certain elliptic differential equations on a hypersphere and their geometric applications, Indiana Univ. Math. J. 28 (1979), 35-51.

33. __ Infinitesimal deformations preserving parallel normal vector fields, Lecture Notes in Math., vol. 792, Springer, 1980, pp. 383-405.

34. - Some remarks on elliptic equations and infinitesimal deformations of submanifolds, Lecture Notes in Math., vol. 838, Springer, 1981, pp. 211-220.

35. C. M. Petty, Centroid bodies, Pacific J. Math. 11 (1961), 1535-1547.

36. _ Projection bodies, Proc. Collog. on Convexity (Copenhagen, 1965), Kobenhavns. Univ. Mat. Inst. 1967, pp. 234-241.

37. A. V. Pogorelov, The Minkowski multidimensional problem, Wiley, New York, 1978.

38. R. Schneider, Zu einem Problem, Projektionen, Körper von Shephard über die projectionen konvexer körper, Math. Z. 101 (1967), 71-82.

39. R. Schneider and W. Weil, Zonoids and related topics, Convexity and Its Applications (P. M. Gruber and J. M. Wills, eds.), Birkhäuser, Basel, 1983, pp. 296-317.

40. R. Schneider, On the Alexandrov-Fenchel inequality, Discrete Geometry and Convexity, (J. E. Goodman et al., eds.), vol. 440, Ann. New York Acad. Sci., 1985, pp. 132-141.

41. J. Schwartz, Nonlinear functional analysis, Gordon and Breach, New York, 1968.

42. L. Schwartz, Theorie des distributions, Hermann, Paris, 1966.

43. R. Strichartz, $L^{p}$ estimates for Radon transforms in Euclidean and non-Euclidean spaces, Duke Math. J. 48 (1981), 699-727.

44. W. Weil, Decomposition of convex bodies, Mathematika 21 (1974), 19-25.

45. Centrally symmetric convex bodies and distributions, Israel J. Math. 24 (1976), 352-367.

46. $\ldots$ Centrally symmetric convex bodies and distributions II, Israel J. Math. 32 (1979), 173-182.

47. __, On surface area measure of convex bodies, Geom. Dedicata 9 (1980), 299-306. 
48. G. Zhang, Intersection bodies and Busemann-Petty inequalities in $\mathbf{R}^{4}$, Ann. of Math. (to appear).

49. - No polytope is an intersection body, (to appear).

50. F. Warner, Foundations of differentiable manifolds and Lie groups, Springer-Verlag, 1983.

51. R. Gardner, A positive answer to the Busemann-Petty problem in three dimensions, Ann. of Math. (to appear).

52. R. Schneider, Convex bodies: The Brunn-Minkowski theory, Cambridge Univ. Press, 1993.

Department of Mathematics, Temple University, Phundelphia, Pennsylvania 19122

E-mail address: gzhangeouclid.math. temple.edu 